\title{
Scattering of ultraviolet radiation in turbid suspensions
}

\author{
Michelle L. Griffith ${ }^{\text {a) }}$ and John W. Halloran \\ Materials Science and Engineering Department, The University of Michigan, Ann Arbor, \\ Michigan 48109-2136
}

(Received 2 November 1995; accepted for publication 10 December 1996)

\begin{abstract}
A Beer's law expression for the penetration depth of ultraviolet radiation in a concentrated suspension of scattering particles is used to model the depth of cure for a suspension of ceramic particles in a medium of photocurable monomers. The cure depth is predominantly controlled by the square of the refractive index difference between the ceramic particles and the medium, $\Delta n^{2}=\left(n_{p}-n_{0}\right)^{2}$. A secondary effect on the cure depth is the ratio of the interparticle spacing to the ultraviolet wavelength. Theoretical results agree with experimental data for $0.40-0.50$ volume fraction ceramic-filled suspensions. (C) 1997 American Institute of Physics.

[S0021-8979(97)06506-7]
\end{abstract}

\section{INTRODUCTION}

The interaction between radiation and particles has been investigated for a variety of phenomena including the scattering of visible light resulting in blue skies and red sunsets. ${ }^{1}$ For particles that are much smaller than the radiation wavelength, scattering behavior is governed by the polarizability tensor, ${ }^{2}$ and particles of simple shape that are much larger than the wavelength may be treated by a combination of geometric optics and Fraunhofer diffraction. ${ }^{3}$ Complex theoretical models are needed to solve the problem for particles of intermediate size. ${ }^{4}$ We consider here the penetration of ultraviolet (UV) radiation in a very concentrated suspension (containing up to 0.50 volume fraction particles) for which no simple expressions are available. ${ }^{2}$ Our particular interest is the case where the particle diameter is similar to the radiation wavelength $(d \propto \lambda)$. For this case, scattering theories describe the interaction of a single object, usually a sphere, suspended in a medium ${ }^{5}$ and cannot be simply applied to concentrated suspensions. For example, the multi-particle scattering equations of $\mathrm{Mie}^{6,7}$ are limited to very dilute suspensions (less than 0.10 solids loading particles), so Mie theory does not accurately describe multiparticle interactions for highly filled suspensions.

It would be advantageous to have a simple expression to describe this radiation interaction. This is particularly relevant for photopolymerization of concentrated suspensions of ceramic powders, which has been used for freeform fabrication of ceramics by stereolithography techniques. ${ }^{8-11}$ Stereolithography produces very accurate three dimensional polymer parts ${ }^{12-14}$ by photopolymerization of a liquid resin with a computer-controlled UV laser. The plastic object is built from a sequence of thin layers about $200 \mu \mathrm{m}$ thick. The depth of cure, $D_{c}$, or the resin polymerized by the UV laser radiation is a critical parameter. A conventional resin is an absorbing medium, and the attenuation of the UV energy with depth can be accurately modeled with Beer's law:

$$
E=E_{0} \exp (-\gamma L)
$$

relating energy density $(E)$ to depth $(L)$ through the extinc-

\footnotetext{
${ }^{a)}$ Present address: Sandia National Laboratories, P.O. Box 5800, MS 0958, Albuquerque, NM 87185; Electronic mail: mlgriff@sandia.gov
}

tion coefficient $(\gamma)$. The cure depth $\left(D_{c}\right)$ is the distance required to attenuate $E$ to the minimum energy density $\left(E_{\text {crit }}\right)$ required for photopolymerization or gellation of the resin, so the cure depth can be described by

$$
D_{c}=[1 / \gamma] \ln \left(E_{0} / E_{\text {crit }}\right) .
$$

Thus the extinction coefficient links cure depth to laser intensity and scan speed and is a vital parameter for stereolithography.

The polymerization depth is reduced by dispersing ceramic powders in a UV-curable medium. For ceramic volume fractions above about 0.10 , the cure depth is controlled by the scattering of the UV radiation. It will be shown that the cure depth of turbid ceramic suspensions can be modeled accurately with an equation of the form:

$$
D_{c}=\frac{2\langle d\rangle}{3 \widetilde{Q}} \frac{n_{0}^{2}}{\Delta n^{2}} \ln \left(\frac{E_{0}}{E_{\text {crit }}}\right),
$$

where $\langle d\rangle$ is the average particle size, $\Delta n^{2}$ is the square of the refractive index difference between the ceramic and the medium $\left[\Delta n^{2}=\left(n_{p}-n_{0}\right)^{2}\right], \phi$ the volume fraction solids, $E_{0}$ the energy density, and $\widetilde{Q}$ is the scattering efficiency term. This equation is a Beer's law expression with an effective extinction coefficient, $\gamma_{\mathrm{eff}}=3 \widetilde{Q} \Delta n^{2} / 2\langle d\rangle n_{0}^{2}$.

This article will cover the general aspects of scattering in relation to particle characteristics, medium characteristics, and regions where scattering or absorption dominate. Theoretical results will be compared to experimental data for highly concentrated ceramic suspensions.

\section{EXPERIMENT}

Details of the experimental work are discussed elsewhere. ${ }^{15,16}$ This article contains the necessary information related to understanding the photopolymerization behavior of highly concentrated ceramic suspensions.

\section{A. Ceramic suspensions}

Ceramic suspensions were made by dispersing ceramic powders in UV-curable solutions. Three ceramic materials were investigated: 
TABLE I. Particle size $(d)$, density, and refractive index $(n)$ for ceramic powders.

\begin{tabular}{|c|c|c|c|c|c|}
\hline Ceramic & $\begin{array}{l}\text { Density } \\
\left(\mathrm{g} / \mathrm{cm}^{3}\right)\end{array}$ & $\begin{array}{c}\text { 50th percentile } \\
\text { particle size } \\
\langle d\rangle(\mu \mathrm{m})\end{array}$ & $\begin{array}{l}\text { 10th percentile } \\
\text { particle size } \\
d_{10}(\mu \mathrm{m})\end{array}$ & $\begin{array}{c}\text { 90th percentile } \\
\text { particle size } \\
d_{90}(\mu \mathrm{m})\end{array}$ & $\begin{array}{l}\text { Refractive } \\
\text { index, } n_{p}\end{array}$ \\
\hline Silica $^{a}$ & 2.65 & $2.29^{\mathrm{e}}$ & 0.79 & 8.70 & 1.56 \\
\hline Silicon nitride ${ }^{b}$ & 3.24 & $0.44^{\mathrm{f}}$ & 0.10 & 0.78 & 2.10 \\
\hline $\begin{array}{l}\text { Alumina } \\
\text { AKP-50 }\end{array}$ & 3.96 & $0.46^{\mathrm{e}}$ & 0.30 & 0.63 & 1.70 \\
\hline $\begin{array}{l}\text { Alumina } \\
\text { AKP-15 }\end{array}$ & 3.96 & $0.61^{\mathrm{e}}$ & 0.41 & 0.93 & 1.70 \\
\hline $\begin{array}{l}\text { Alumina }^{d} \\
\text { RC-HP }\end{array}$ & 3.96 & $0.34^{\mathrm{f}}$ & 0.18 & 0.60 & 1.70 \\
\hline
\end{tabular}

${ }^{\mathrm{a}} \alpha$-quartz, Atlantic Equipment Engineering, Bergenfield, NJ.

bUBE E10, UBE Industries, Tokyo, Japan.

${ }^{\mathrm{c} A K P}-15$ and AKP-50 grades, Sumitomo Chemical, Tokyo, Japan.

${ }^{\mathrm{d}}$ Reynolds RC-HP, Malakoff Industries, Malakoff, TX.

${ }^{\mathrm{e}}$ MICROTRAC particle size analyzer, Leeds \& Northrop, North Wales, PA.

${ }^{\mathrm{f}}$ Particle size distribution analyzer, Model \#CAPA-700, Horiba, Irvine, CA.

(1) silica, a low refractive index ceramic for investment casting applications,

(2) alumina, with a medium refractive index for structural ceramics, and

(3) silicon nitride, a high refractive index material also used for structural ceramic applications.

Table I shows the particle size, density, and refractive index for these ceramic powders. ${ }^{17,18}$

Two varieties of UV-curable solutions were prepared:

(1) aqueous solutions using acrylamide monomers, similar to the "gel casting" formulation ${ }^{19,20}$ with the thermal initiators replaced by photoinitiators, and

(2) a low viscosity diacrylate monomer, hexane diol diacrylate, similar to existing monomer resins used in stereolithography.

Table II shows the refractive index values for the constituents and for the different UV-curable solutions (measured by Abbey refractometry). It was possible to vary the refractive index of the aqueous solutions by the addition of ethylene glycol as a solvent.

For photopolymerization to occur, small amounts of photoinitiators, less than $2.0 \mathrm{wt} \%$, were added to the UVcurable solution. The photoinitiators were matched to the long ultraviolet wavelengths $(\lambda>300 \mathrm{~nm})$, and the concentration chosen for the maximum depth of cure. ${ }^{21}$ After preparing the UV-curable solution, ceramic powder was added, incrementally, and mixed in a high shear mixer. After reach-

TABLE II. Refractive indices for UV-curable solutions and the basic constituents.

\begin{tabular}{cclc}
\hline $\begin{array}{c}\text { Basic materials } \\
\text { for UV-curable solution }\end{array}$ & $\begin{array}{c}\text { Refractive } \\
\text { index, } n\end{array}$ & $\begin{array}{c}\text { UV-curable } \\
\text { solution }\end{array}$ & $\begin{array}{c}\text { Refractive index, } \\
n_{0}\end{array}$ \\
\hline acrylamide & 1.53 & Aqueous 1 & 1.38 \\
methylene bisacrylamide & 1.52 & Aqueous 2 & 1.40 \\
ethylene glycol & 1.43 & Aqueous 3 & 1.41 \\
water & 1.33 & Aqueous 4 & 1.42 \\
& & Aqueous 5 & 1.44 \\
& & Diacrylate & 1.46 \\
\hline \hline
\end{tabular}

ing the maximum solids loading, the photoinitiators were added and the suspension mixed for approximately $5 \mathrm{~h}$ to ensure complete homogenization.

\section{B. Ultraviolet exposure}

Each suspension was exposed to ultraviolet radiation from either a mercury lamp or a laser. The two strongest lines emitted by the lamp, 313 and $366 \mathrm{~nm}$, correspond closely to the wavelengths used in stereolithography machines, $312 \mathrm{~nm}$ for $\mathrm{He}-\mathrm{Cd}$ and $351 \mathrm{~nm}$ for $\mathrm{Ar}-$ ion laser. Cure depths were similar whether exposed to the lamp or the laser. ${ }^{16}$ Exposure doses $\left(\mathrm{mJ} / \mathrm{cm}^{2}\right)$ were measured using a radiometer. After exposure, the polymerized ceramic film was removed from the remaining material, rinsed, and the cure depth was measured either by optical or electron microscopy.

\section{Experimental results}

\section{Concentration dependence}

Figures 1(a) and 1(b) show the concentration dependence on the cure depth for silica, alumina, and silicon nitride dispersed in a variety of UV-curable solutions. At 0.50 solids loading for the silica and alumina suspensions, the cure depth is greater than $200 \mu \mathrm{m}$, thereby satisfying the requirements for use in stereolithography.

The cure depth, $D_{c}$, is plotted against the inverse of volume fraction solids, $1 / \phi$, showing linear behavior. Even though a 0.50 volume fraction silicon nitride/diacrylate suspension was not prepared, the cure depth is predicted to equal $40 \mu \mathrm{m}$. The largest cure depths are for the silica suspensions, for which the square of the refractive index difference, $\Delta n^{2}$, has the smallest value. However, at 0.50 solids loading, the cure depth for the alumina and silica suspensions are similar.

\section{Dose dependence}

Figures 2(a) and 2(b) show the dose dependence for 0.50 solids loading ceramic suspensions. As expected, the cure depth is larger as the dose increases, following Beer's law and the cure depth equation, $D_{c}$. Note that the cure depth 

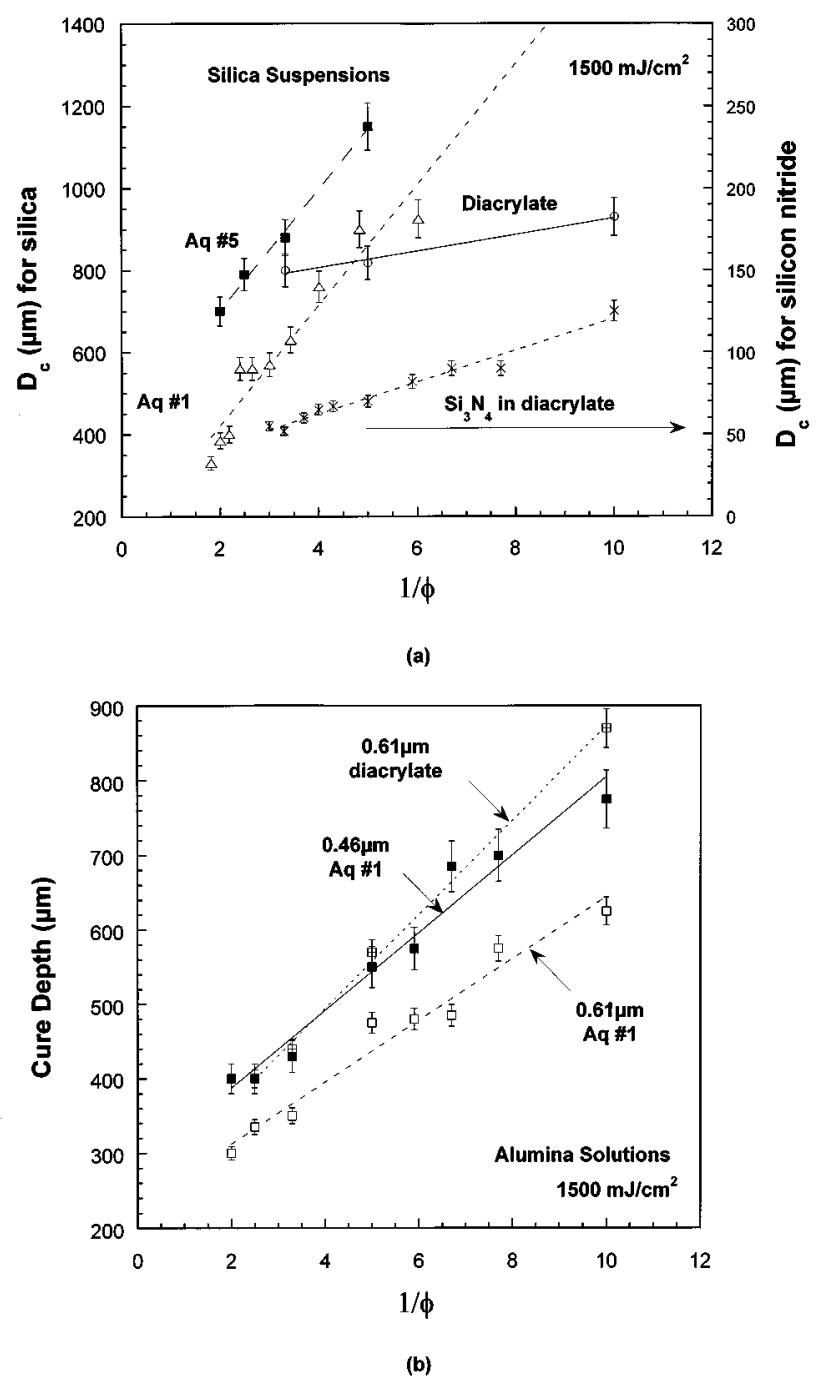

FIG. 1. (a) Depth of cure $\left(D_{c}\right)$ vs inverse volume fraction $(1 / \phi)$ for three silica suspensions and for silicon nitride dispersed in diacrylate. All samples received an exposure dose of $1500 \mathrm{~mJ} / \mathrm{cm}^{2}$. (b) Depth of cure $\left(D_{c}\right)$ vs inverse volume fraction $(1 / \phi)$ for three alumina suspensions. All suspensions received an exposure dose of $1500 \mathrm{~mJ} / \mathrm{cm}^{2}$.

greatly increases as the square of the difference in the refractive index between silica and the aqueous UV-curable solution is reduced. At $1500 \mathrm{~mJ} / \mathrm{cm}^{2}$, the cure depth changes from 250 to $700 \mu \mathrm{m}$ as $\Delta n^{2}$ changes from 0.032 to 0.014 .

\section{Particle size dependence}

Figure 1(b) shows the cure depth versus $1 / \phi$ for different particle size alumina powders dispersed in a variety of UVcurable solutions. All suspensions have a linear fit, following the expected cure depth behavior. However, the smaller particle size alumina has a larger cure depth in the aqueous solution which is contradictory to the expectation that $D_{c} \propto d$.

\section{THE SCATTERING EQUATION $D_{c}$}

Scattering theory is based upon an electromagnetic wave impinging on a single particle, with a particular refractive index and size, in a medium with a different refractive index. ${ }^{2,5}$ Most theories assume that the medium does not

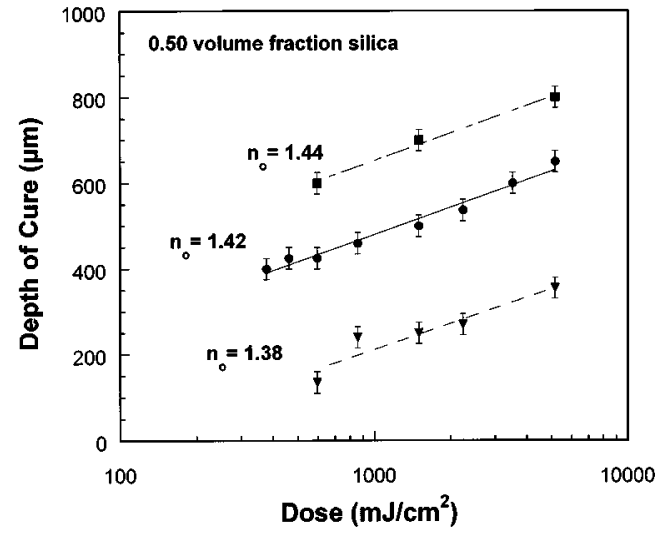

(a)

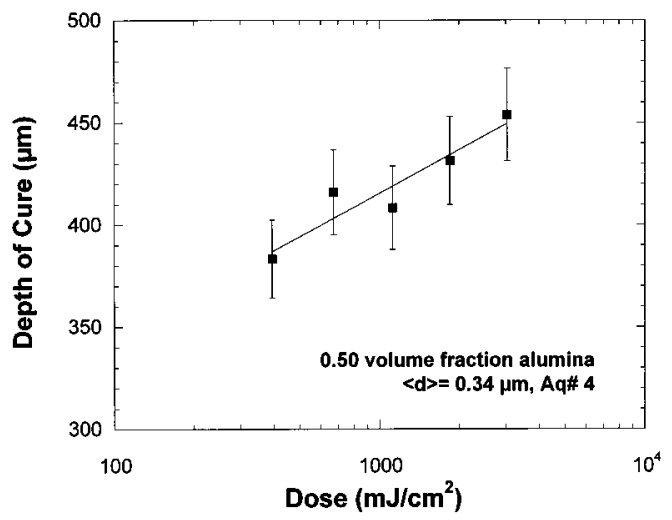

(b)

FIG. 2. Depth of cure $(\mu \mathrm{m})$ vs dose $\left(\mathrm{mJ} / \mathrm{cm}^{2}\right)$ for 0.50 volume fraction silica in three aqueous UV-curable solutions. (b) Depth of cure $(\mu \mathrm{m})$ vs dose $\left(\mathrm{mJ} / \mathrm{cm}^{2}\right)$ for 0.50 volume fraction alumina dispersed in aqueous solution \#4.

absorb the radiation, and for turbid suspensions the scattering properties will dominate the penetration depth.

For multi-particle scattering, a form of Beer's law is utilized where the particles act as separate scattering centers and the scattering term is a summation of the individual scattering centers. For the summation to be valid, the distance between particles must be greater than two radii which corresponds to suspensions at solid loadings of less than 0.10 when using particles on the order of $1 \mu \mathrm{m}$. However, there are spaces between particles which are roughly one particle radius, and therefore the summation form for multi-particle scattering will be used without much loss in validity. The general form of Beer's law is

$$
E=E_{0} \exp (-\gamma L),
$$

where $E_{0}$ is the energy density (measured in $\mathrm{mJ} / \mathrm{cm}^{2}$ ) of the radiation at the surface of suspension, $\gamma$ is the extinction coefficient or turbidity term, and $L$ is the path length the radiation travels.

Two processes occur as the UV radiation travels into the highly concentrated suspension. First, and predominantly, the particles scatter the radiation. Second, the UV-curable solution absorbs part of the radiation, thereby reducing its intensity. The radiation which is not absorbed travels further into the suspension and the two processes continue until the energy of the radiation reaches a critical limit, $E_{\text {crit }}$, in which 
the remaining radiation is absorbed by the monomer resin. This minimum energy is required for gellation of the monomer. One can describe the polymerization depth for a turbid ceramic suspension, where the path length equals the depth of cure, $L \rightarrow D_{c}$, and the exposure equals a critical exposure, $E \rightarrow E_{\text {crit }}$, where

$$
E_{\text {crit }}=E_{0} \exp \left(-\gamma D_{c}\right), \text { or } D_{c}=\gamma^{-1} \ln \left(\frac{E_{0}}{E_{\text {crit }}}\right) \text {. }
$$

The extinction coefficient can be described by ${ }^{22}$

$$
\gamma=\frac{3}{2} \frac{\phi Q}{\langle d\rangle},
$$

where $\langle d\rangle$ is the average particle diameter, $\phi$ is the volume fraction particles, and $Q$ is the extinction coefficient efficiency.

After substitution, the scattering equation becomes

$$
D_{c}=\frac{2\langle d\rangle}{3 Q} \frac{1}{\phi} \ln \left[\frac{E_{0}}{E_{\text {crit }}}\right] \text {. }
$$

Equation 2 exhibits three linear proportional relationships expected for highly turbid suspensions:

(1) the cure depth versus $1 / Q$ at fixed volume fraction and $\langle d\rangle$,

(2) the cure depth versus $1 / \phi$ at fixed $Q$ and $\langle d\rangle$, and

(3) the cure depth versus $\ln \left(E_{0}\right)$ at fixed $\phi,\langle d\rangle$, and $Q$. (The curves of $D_{c}$ vs $\log \left(E_{0}\right)$ and $D_{c}$ vs $1 / \phi$ show that the behavior of highly concentrated ceramic suspensions follows the expected relationships in Eq. (2), provided that $Q$ does not depend on $\phi$.)

For scattering in highly turbid suspensions, the influence of the extinction coefficient efficiency, volume fraction solids, and exposure dose on the cure depth will be determined for the ceramic suspensions in this work, therefore aiding in the prediction of cure depth for any UV-curable ceramic suspension.

\section{THE EXTINCTION COEFFICIENT EFFICIENCY $Q$}

The dimensionless function $Q$ embodies a complex physics for scattering behavior in a dense system. In general, one might expect $Q$ to depend on $\phi,\langle d\rangle$, and the ratio $m=n_{p} / n_{0}$, with $^{2}$

$Q(\phi, x, m)=(m-1)^{2} \widetilde{Q}(\phi, x, m)=\left(\Delta n / n_{0}\right)^{2} \widetilde{Q}(\phi, x, m)$,

where $x=\pi\langle d\rangle / \lambda, n_{p}$ is the particle refractive index, $n_{0}$ is the UV-curable solution refractive index, and $\Delta n$ is the difference between the two.

There are several closed-form equations for $Q^{23-25}$ in terms of $x$ and $\rho$, where $\rho$ is related to the refractive index difference between the ceramic and the UV-curable solution

$$
\rho=x \Delta n=x\left(n_{p}-n_{0}\right) .
$$

For the present case, the particle size is equal to or greater than the wavelength of radiation, so Rayleigh scattering is not appropriate for $Q .{ }^{23}$
TABLE III. Refractive index dependence on the scattering efficiency term $Q$ for turbid silica suspensions; a comparison between the experimental value and three theoretical values. Values used in Eq. (2) for $Q_{\exp }:\langle d\rangle$ $=2.29 \mu \mathrm{m}, n_{p}=1.56$ (at $\left.\lambda=366 \mathrm{~nm}\right), E_{0}=1500 \mathrm{~mJ} / \mathrm{cm}^{2}, E_{\text {crit }}=20 \mathrm{~mJ} / \mathrm{cm}^{2}$, $\phi=0.50$.

\begin{tabular}{ccccccc}
\hline \hline $\begin{array}{c}\text { UV-curable } \\
\text { solution }\end{array}$ & $n_{0}$ & $D_{c}(\mu \mathrm{m})$ & $Q_{\exp }$ & $Q_{\text {ray }}$ & $Q_{\text {mie }}$ & $Q_{n}$ \\
\hline Aq \#1 & 1.38 & 250 & 0.053 & 6.09 & 2.12 & 0.017 \\
Aq \#2 & 1.40 & 340 & 0.039 & 5.00 & 1.82 & 0.013 \\
Aq \#3 & 1.41 & 450 & 0.030 & 4.06 & 2.16 & 0.011 \\
Aq \#4 & 1.42 & 490 & 0.027 & 3.64 & 2.00 & 0.010 \\
Aq \#5 & 1.44 & 700 & 0.019 & 2.42 & 2.20 & 0.007 \\
diacrylate & 1.46 & 760 & 0.017 & 2.03 & 2.03 & 0.005 \\
\hline \hline
\end{tabular}

In the Rayleigh-Gans region, $Q_{\text {ray }}$ is a strong function of $x$ and the square of the refractive index difference, $\Delta n^{2}$, as shown in the equation below:

$$
Q_{\text {ray }}=\frac{1}{2} \rho^{2}=\frac{1}{2} x^{2} \Delta n^{2} .
$$

However, for $Q_{\text {ray }}$ to be valid, the term $\rho$ must be $\ll 1$, corresponding to a small difference in the refractive indices: scattering occurs but not to a significant degree. Therefore, $Q$ must be much less than 1 .

The particle size and refractive index difference can be very large for the case of Mie scattering, where

$$
Q_{\mathrm{mie}}=2-\frac{4 \sin \rho}{\rho}+\frac{4}{\rho^{2}}(1-\cos \rho) .
$$

Nevertheless, the overall magnitude of $Q_{\text {mie }}$ is small. The Mie $Q$ is an oscillating function of $\rho$, where for large $x, Q_{\text {mie }}$ approaches a value of 2 .

Theoretical forms for $Q$ will be compared to experimental results using Eq. (2). By determining the scattering behavior through the term $Q$, the cure depth behavior for turbid ceramic suspensions will be understood. Only the silica and alumina suspensions will be discussed in detail. Results and discussions for these suspensions can be applied to the silicon nitride suspension.

\section{A. The function $Q$ for silica suspensions}

\section{Dependence on refractive index of the UV-curable solution}

The extinction coefficient efficiency $Q$ embodies the scattering behavior for turbid suspensions. In Table III, experimental values for $Q$ are compared with calculated forms for all silica-UV solutions. An additional form for $Q$, similar to Eq. (3), will be considered, in which the predominant behavior is a scaling with the square of the refractive index difference, or $Q_{n}=\Delta n^{2} / n_{0}^{2}$. The average particle size is used even though the silica powder has a wide particle size distribution; a more rigorous treatment of particle size effects will not change the overall trends in the cure depth behavior. ${ }^{23}$ The value for $E_{\text {crit }}$ was set to $20 \mathrm{~mJ} / \mathrm{cm}^{2}$ for all UV-curable solutions. $^{26-28}$

Looking at Fig. 2(a), a linear fit is expected as $\Delta n^{2}$ decreases (e.g., $D_{c} \uparrow$ as $\Delta n^{2} \downarrow$ ). Forms of $Q$, which expect a large influence on the square of the refractive index difference, $Q_{\text {ray }}$ and $Q_{n}$, show the expected linear behavior where 
TABLE IV. Dose dependence on the scattering efficiency term $Q$ for a turbid silica suspension; a comparison between the experimental value and three theoretical values. Values used in Eq. (2) for $Q_{\exp }:\langle d\rangle=2.29 \mu \mathrm{m}$, $n_{p}=1.56($ at $\lambda=366 \mathrm{~nm}), E_{\text {crit }}=20 \mathrm{~mJ} / \mathrm{cm}^{2}, \phi=0.50, n_{0}=1.42($ at $\lambda=366$ $\mathrm{nm})$.

\begin{tabular}{cccccc}
\hline \hline $\begin{array}{c}E_{0} \\
\left(\mathrm{~mJ} / \mathrm{cm}^{2}\right)\end{array}$ & $D_{c}(\mu \mathrm{m})$ & $Q_{\text {exp }}$ & $Q_{\text {ray }}$ & $Q_{\text {mie }}$ & $Q_{n}$ \\
\hline 380 & 400 & 0.023 & & & \\
600 & 425 & 0.024 & & & \\
860 & 460 & 0.025 & & & \\
1500 & 490 & 0.027 & 3.64 & 2.00 & 0.010 \\
2200 & 540 & 0.027 & & & \\
5100 & 630 & 0.027 & & & \\
\hline \hline
\end{tabular}

$D_{c}$ is proportional to $1 / Q$ or $1 / \Delta n^{2}$. However, the square of the refractive index difference form of $Q, Q_{n}$, best matches the experimental results as shown in Table III. General observations show the Rayleigh-Gans equation for $Q$ is not strictly valid since the values are greater than one. Mie theory will not produce a linear fit if the cure depth is plotted against $1 / Q_{\text {mie }}$. So the square of the refractive index difference is the major factor influencing the depth of cure for turbid suspensions and the particle size term $x^{2}$ must be a minor influence given that the Rayleigh-Gans form for $Q$ does not explain the scattering behavior.

\section{Dependence on dose}

From Fig. 2(a), the experimental extinction coefficient, $Q_{\text {exp }}$, can be determined and compared with the three theoretical forms: $Q_{\text {ray }}, Q_{\text {mie }}$, and $Q_{n}$. Table IV shows the results for the aqueous UV-curable solution with a refractive index of 1.42. Once again, the square of the refractive index difference form of $Q$ best matches the experimental results, where $Q_{\exp }$ ranges from 0.023 to 0.027 , and $Q_{n}=0.010$.

The silica suspensions are best described by the square of the refractive index difference form of the scattering efficiency, $Q_{n}$, where $Q_{n}=\Delta n^{2} / n_{0}^{2}$.

\section{B. The term $Q$ for alumina suspensions}

\section{Dependence on refractive index of the UV-curable solution}

From Table $\mathrm{V}$, the magnitude of the scattering efficiency term, $Q_{n}\left(Q=\Delta n^{2} / n_{0}^{2}\right)$, best matches the experimental results for a variety of UV-curable solutions. Even though the square of the refractive index difference, $\Delta n^{2}$, best describes the scattering behavior, it does not determine the effect of
TABLE VI. Dose dependence on the scattering efficiency term $Q$ for a turbid alumina suspension; a comparison between the experimental value and three theoretical values. Values used for $Q_{\exp }:\langle d\rangle=0.34 \mu \mathrm{m}, n_{p}=1.71$ (at $\lambda=366 \mathrm{~nm}), E_{\text {crit }}=20 \mathrm{~mJ} / \mathrm{cm}^{2}, \phi=0.50, n_{0}=1.42$ (at $\left.\lambda=366 \mathrm{~nm}\right)$.

\begin{tabular}{cccccc}
\hline \hline $\begin{array}{c}E_{0} \\
\left(\mathrm{~mJ} / \mathrm{cm}^{2}\right)\end{array}$ & $D_{c}(\mu \mathrm{m})$ & $Q_{\text {exp }}$ & $Q_{\text {ray }}$ & $Q_{\text {mie }}$ & $Q_{n}$ \\
\hline 390 & 381 & 0.0035 & & & \\
660 & 402 & 0.0039 & & & \\
1100 & 417 & 0.0044 & 0.34 & 2.19 & 0.042 \\
1800 & 430 & 0.0047 & & & \\
2900 & 452 & 0.0050 & & & \\
\hline \hline
\end{tabular}

particle size. With Eq. (2) and assuming $Q=\Delta n^{2} / n_{0}^{2}$, it is expected that the cure depth is proportional to the particle size, where $D_{c} \propto\langle d\rangle$. However, the results in Fig. 1(b) show the opposite effect. The term $Q$ must include particle size effects on cure depth behavior in turbid suspensions.

\section{Dependence on dose}

From Fig. 2(b), the experimental coefficient efficiency term can be determined and compared with the three theoretical forms as shown in Table VI. Once again, the square of the refractive index difference form, $Q_{n}=\Delta n^{2} / n_{0}^{2}$, best matches the experimental results.

The alumina results show that $Q_{n}$ best describes the scattering behavior for turbid suspensions but does not accurately describe the particle size effect.

\section{THE $\tilde{Q}$ TERM}

With an understanding that the scattering efficiency $Q_{n}$ best describes the magnitude of the cure depth $D_{c}$, it is necessary to discover other factors which influence the cure depth behavior through the term $\widetilde{Q}$ in Eq. (3), where

$$
Q_{\exp }=\frac{2 d}{3 \phi D_{c}} \ln \left(\frac{E_{0}}{E_{\text {crit }}}\right)=Q=\widetilde{Q} \frac{\Delta n^{2}}{n_{0}^{2}},
$$

or

$$
\widetilde{Q}=\frac{n_{0}^{2}}{\Delta n^{2}} \frac{2 d}{3 \phi D_{c}} \ln \left(\frac{E_{0}}{E_{\text {crit }}}\right) .
$$

The term $\widetilde{Q}$ may be a function of the volume fraction $(\phi)$, the particle size $(x)$, the dose $\left(E_{0}\right)$, and the square of the refractive index difference $\left(\Delta n^{2}\right)$; its behavior as a function of these variables will be determined.

TABLE V. Refractive index and particle size dependence on the scattering efficiency term $Q$ for turbid alumina suspensions; a comparison between the experimental value and three theoretical values. Values used for $Q_{\exp }$ : $n_{p}=1.71($ at $\lambda=366 \mathrm{~nm}), E_{0}=1500 \mathrm{~mJ} / \mathrm{cm}^{2}, E_{\text {crit }}=20 \mathrm{~mJ} / \mathrm{cm}^{2}$.

\begin{tabular}{cccccccccc}
\hline \hline \multirow{2}{*}{ Alumina } & $\begin{array}{c}\text { UV-curable } \\
\text { solution }\end{array}$ & $n_{0}$ & $\begin{array}{c}\langle d\rangle \\
(\mu \mathrm{m})\end{array}$ & $\phi$ & $D_{c}(\mu \mathrm{m})$ & $Q_{\exp }$ & $Q_{\text {ray }}$ & $Q_{\text {mie }}$ & $Q_{n}$ \\
\hline AKP-50 & Aq \#1 & 1.38 & 0.46 & 0.5 & 400 & 0.007 & 0.78 & 2.2 & 0.057 \\
AKP-15 & Aq \#1 & 1.38 & 0.61 & 0.5 & 300 & 0.012 & 1.36 & 2.93 & 0.057 \\
RC-HP & Aq \#4 & 1.42 & 0.34 & 0.5 & 450 & 0.004 & 0.34 & 2.19 & 0.042 \\
RC-HP & Diacrylate & 1.46 & 0.34 & 0.4 & 275 & 0.009 & 0.25 & 0.9 & 0.029 \\
AKP-15 & Diacrylate & 1.46 & 0.61 & 0.5 & 370 & 0.010 & 0.81 & 2.24 & 0.029 \\
\hline \hline
\end{tabular}




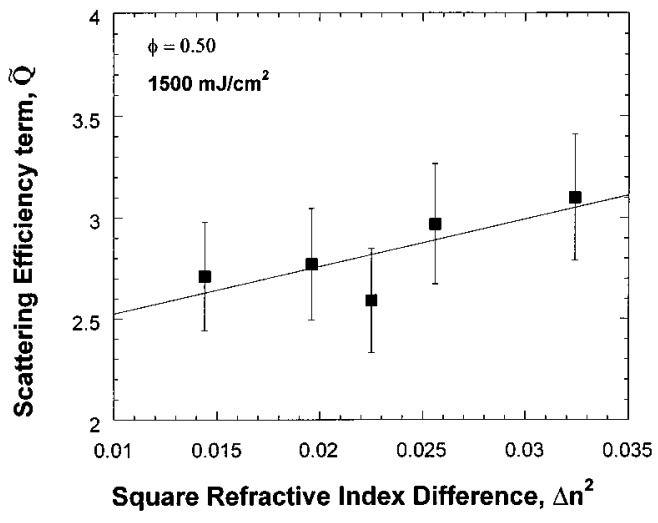

FIG. 3. The experimental scattering efficiency term $\widetilde{Q}$ as a function of the refractive index difference $\left(\Delta n^{2}\right)$ for 0.50 solids loading silica in the five aqueous solutions.

\section{A. Experimental values for silica suspensions}

\section{Refractive index difference dependence}

Figure 3 shows the influence of refractive index difference on the term $\widetilde{Q}$. Looking at the data for high solids aqueous silica suspensions, the value for $\widetilde{Q}$ varies between 2.5 and 3.0. The value rises slightly as the refractive index difference increases, or where more scattering behavior is expected.

\section{Volume fraction dependence}

Fig. 4 results in two general behaviors (the influence of volume fraction on the term $\widetilde{Q}$ ). Both curves show linear behavior, but the addition of solids in the UV-curable suspension with a refractive index of 1.44 (Aq\#5) strongly affects the value for $\widetilde{Q}$. Since the square of the refractive index difference, $\Delta n^{2}$, decreases by $50 \%$ in comparison to Aq\#1, there must be a transition from absorption to scattering dominated behavior as the solids content increases. This will be discussed in a later section. Note, at high solids contents, the value for $\widetilde{Q}$ varies between 2 and 3 , similar to the results for the refractive index difference dependence.

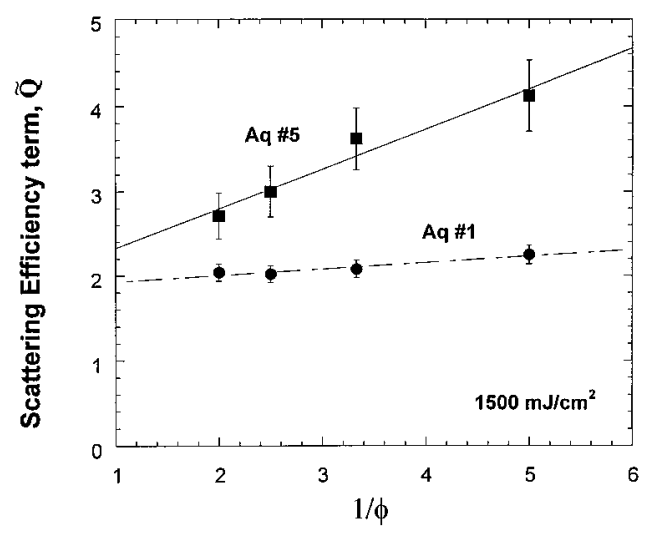

FIG. 4. The experimental or expected scattering efficiency term $\widetilde{Q}$ as a function of volume fraction $(1 / \phi)$ for silica dispersed in two aqueous UVcurable solutions (Aq \#1 and Aq \#5).

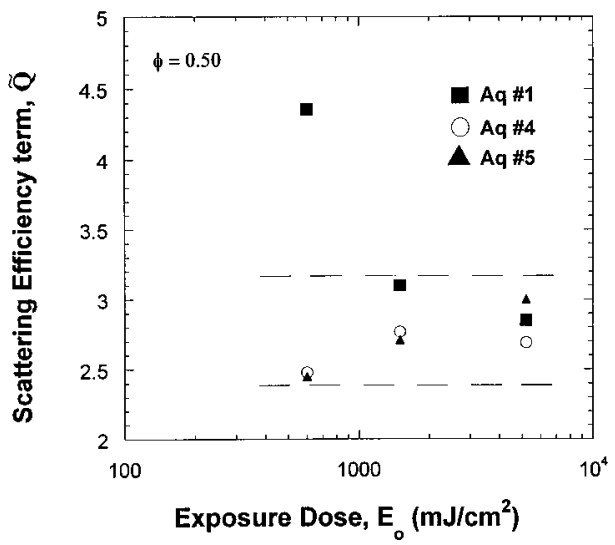

FIG. 5. The experimental scattering efficiency term $\widetilde{Q}$ as a function of the exposure dose $\left(E_{0}\right)$ for 0.50 solids loading silica in three aqueous solutions.

\section{Dose dependence}

In Fig. 5, the dose dependence on the term $\widetilde{Q}$ has similar results, where the value varies between 2.5 and 3.25. At one low dose, there is an inconsistent data point for silica in the UV-curable aqueous suspension with a refractive index of 1.38 (Aq\#1). Possibly the cure depth was measured incorrectly or the polymerization behavior is quite different at low doses in this highly scattering suspension; the rest of the data for three high solids loading aqueous suspensions show similar results.

By looking at three conditions for silica suspensions, the value for $\widetilde{Q}$ lies between 2 and 3 .

\section{B. Experimental values for alumina suspensions}

\section{Refractive index difference dependence}

The effect of the refractive index on the term $\widetilde{Q}$ will be discussed in relation to the particle size of alumina dispersed in the UV-curable suspensions. Table VII shows the experimental values for $\widetilde{Q}$ in relation to the refractive index difference for three 0.50 solids loading suspensions. The value of $\widetilde{Q}$ ranges between 0.131 and 0.238 . As expected, the value for $\widetilde{Q}$ decreases when smaller particle size alumina is used in the same suspension $\left(D_{c} \propto 1 / \widetilde{Q}\right)$.

\section{Volume fraction dependence}

Figure 6 shows the dependence of volume fraction on $\widetilde{Q}$, where at high solids loading, the value varies between 0.12 and 0.35 for the three solutions discussed in the previous

TABLE VII. Experimental values for $\widetilde{Q}$ as a function of the refractive index difference and particle size. Values used for $\widetilde{Q}: n_{p}=1.71$ (at $\lambda=366 \mathrm{~nm}$ ), $E_{0}=1500 \mathrm{~mJ} / \mathrm{cm}^{2}, \phi=0.50$.

\begin{tabular}{cccccc}
\hline \hline Alumina & $\begin{array}{c}\text { UV-curable } \\
\text { solution }\end{array}$ & $n_{0}$ & $\begin{array}{c}\langle d\rangle \\
(\mu \mathrm{m})\end{array}$ & $D_{c}(\mu \mathrm{m})$ & $\widetilde{Q}_{\text {exp }}$ \\
\hline AKP-50 & Aq \#1 & 1.38 & 0.46 & 400 & 0.134 \\
AKP-15 & Aq \#1 & 1.38 & 0.61 & 300 & 0.238 \\
RC-HP & Aq \#4 & 1.42 & 0.34 & 420 & 0.131 \\
\hline \hline
\end{tabular}




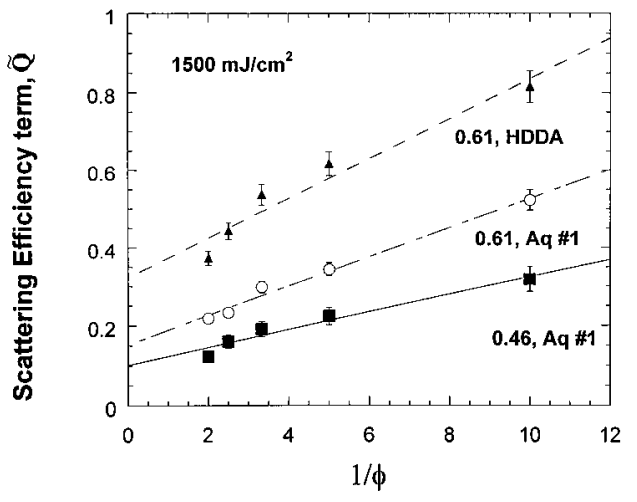

FIG. 6. The experimental scattering efficiency term $\widetilde{Q}$ as a function of volume fraction $(1 / \phi)$ for alumina dispersed in three UV-curable solutions.

section. All solutions have a strong dependence on volume fraction, where the scattering increases as more particles are added to the suspension.

\section{Dose dependence}

For alumina powder with an average particle size of 0.34 $\mu \mathrm{m}$ dispersed at a solid loading of 0.50 in the aqueous suspension with a refractive index of $1.42(\mathrm{Aq \# 4})$, there is an increasing value for $\widetilde{Q}$ as the exposure dose increases as shown in Fig. 7. The value of $\widetilde{Q}$ varies between 0.080 and 0.140 as the exposure dose increases from 400 to 4000 $\mathrm{mJ} / \mathrm{cm}^{2}$.

The alumina suspensions show a wider range of values for $\widetilde{Q}$, which is expected for the variance in the particle sizes, where $0.10<\widetilde{Q}<0.35$.

\section{C. $\tilde{Q}$ as a function of particle size}

Without taking the particle size into account, $Q$ (from $Q_{n}$ ) predicted the scattering behavior of the silica solutions, but did not accurately describe the alumina data. The results for alumina show as the particle size became finer, the cure depth increased. In contrast, Eq. (2), when $Q=\Delta n^{2} / n_{0}^{2}$ is substituted, predicts the cure depth should increase with increasing particle size.

For the extinction coefficient efficiency equation, $Q=\widetilde{Q} \Delta n^{2} / n_{0}^{2}$, the term $\widetilde{Q}$ is expected to contain the particle

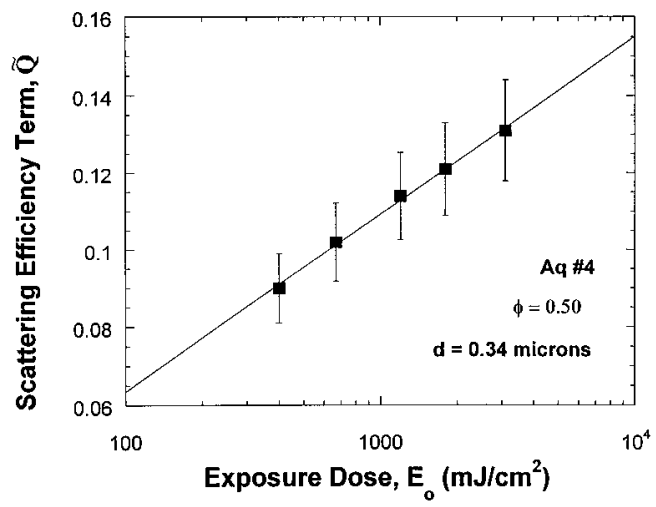

FIG. 7. The experimental scattering efficiency term $\widetilde{Q}$ as a function of the exposure dose $\left(E_{0}\right)$ for 0.50 solids loading alumina in aqueous solution \#4.
TABLE VIII. Comparison of the experimental value for $\widetilde{Q}$ to the calculated value.

\begin{tabular}{ccccc}
\hline \hline & $\begin{array}{c}\text { Experimental } \\
\text { value }\end{array}$ & & Interparticle & $\begin{array}{c}\text { Calculated } \\
\text { value }\end{array}$ \\
Ceramic & $\widetilde{Q}_{\exp }$ & $\langle d\rangle(\mu \mathrm{m})$ & $\begin{array}{c}\text { Inacing, } S(\mathrm{~nm}) \\
\widetilde{Q}=S / \lambda_{0}\end{array}$ \\
\hline silica & $2<\widetilde{Q}<3$ & 2.29 & 949 & 2.59 \\
alumina & $0.10<\widetilde{Q}<0.35$ & 0.61 & 253 & 0.69 \\
& & 0.46 & 191 & 0.52 \\
silicon nitride & 0.68 & 0.34 & 144 & 0.39 \\
\hline \hline
\end{tabular}

size-wavelength relationship and its effect on the depth of cure. ${ }^{2,5}$ However, the behavior of turbid suspensions could not be described when the particle size was related directly to the wavelength through the term $x$ in the Rayleigh-Gans and Mie formulas for $Q$. Assuming that the scattering coefficiency term $Q$ is related to $d / \lambda$ is similar to scattering theories that consider one particle in a medium. ${ }^{2,5}$ Experimental results of turbid suspensions show that scattering is dominated by the refractive index difference, not by the particle size. Moreover, at the volume fractions considered in turbid suspensions, the relationship to be considered is the interparticle spacing, not the particle size. The spacing between particles will affect how the radiation penetrates the suspension, where interference effects as well as absorption by the photoinitiator(s) determine the cure distance. This interference phenomenon is complicated ${ }^{29,30}$ and cannot be described with simple formulas. With radiation traveling from a variety of directions through an array of finely spaced particles, the resulting interference pattern is difficult to describe and beyond the scope of basic theory.

Using simple cubic packing, the median particle size for ceramics in this study, and a 0.50 solids loading, the interparticle spacing, $S$, is calculated in Table VIII. For the silica suspensions with a large particle size, the interparticle spacing is $950 \mathrm{~nm}$, which is several times larger than the UV wavelength. For the alumina suspensions using fine particles, the spacing varies from 140 to $250 \mathrm{~nm}$ which is less than the UV wavelength. With $\widetilde{Q} \propto S / \lambda$ and $\lambda=366 \mathrm{~nm}, \widetilde{Q}>1$ for the silica suspensions (coarse powder), and $\widetilde{Q}<1$ for the alumina suspensions (fine powders).

Also shown in Table VIII, are the experimental values for $\widetilde{Q}$ from the data using $Q$. The value of $\widetilde{Q}$ is difficult to understand in terms of $d / \lambda$, but in terms of $S / \lambda$, turbid suspensions can be described by Eq. (2). For example, the silica suspensions are described by Eq. (2), when the value of $\widetilde{Q}$ is between 2 and 3. With $\widetilde{Q}=S / \lambda$, the value of $\widetilde{Q}=2.59$.

In the case of alumina, the value of $\widetilde{Q}$ must be roughly between 0.10 and 0.35 for 0.50 solids loading suspensions, and with $\widetilde{Q}=S / \lambda$, the calculated range is $0.39<\widetilde{Q}<0.69$. This reasonably describes the alumina suspensions, where the interparticle spacing dominates over particle size in predicting the depth of cure. Moreover, the difference in cure depth related to particle size can be explained using the particle spacing, $S$, where smaller particle size results in a smaller $\widetilde{Q}$ value, and therefore the cure depth increases. 
Even though the silicon nitride data has not been discussed, Table VIII shows that $\widetilde{Q}=S / \lambda$ reasonably describes the scattering behavior for this highly turbid suspension. Note, the square of the refractive index difference, $\Delta n^{2}$, is still the major determinant for the magnitude of the depth of cure.

\section{DISCUSSION}

\section{A. Quantitative prediction of $\boldsymbol{D}_{c}$}

With $Q=\Delta n^{2} / \Delta n_{0}^{2}$, the magnitude of the cure depth can be predicted for high solids loading turbid suspensions. However, by looking at the curves in Figs. 5 and 7, there is a noticeable effect that the dose has on the value $\widetilde{Q}$. Moreover, the volume fraction dependence shows different behaviors in Figs. 4 and 6 . The theoretical equation for turbid suspensions describes the cure depth behavior well, but an absorption term should be included, where the absorption term would describe behaviors related to the absorption limited region and/or dilute limit in these suspensions and is dependent upon the photochemistry of the UV-curable solution. To determine this behavior, polymerization research at low volume fractions must be investigated.

We propose a general scattering equation that describes the cure behavior for nonabsorbing ceramic powders dispersed in ultraviolet curable solutions:

$$
D_{c}=\frac{2\langle d\rangle}{3 \widetilde{Q}} \frac{n_{0}^{2}}{\Delta n^{2}} \ln \left(\frac{E_{0}}{E_{\text {crit }}}\right),
$$

where

$$
\widetilde{Q} \propto \frac{S}{\lambda} .
$$

This model of scattering behavior is a simple model for understanding the magnitude of the cure depth for turbid suspensions where absorption does not play a major role. Refinement of the model is best handled, first by the addition of an absorption term. Further physical understanding of the cure depth behavior should consider more complex theories. For example, diffuse wave spectroscopy ${ }^{30}$ is not constrained to low volume fractions for the description of radiation traveling through a suspension. This theory can describe the scattering behavior of concentrated suspensions, but needs to include an absorption term for the polymerization of the medium. Further research will refine the basic model in this work and incorporate other theories.

\section{B. Predicting cure depth for other ceramics}

Figure 8 shows the refractive index versus density for a variety of ceramic materials. Superimposed on the graph are the refractive indices for water, the diacrylate, and methyl naphthalene. Water represents a low refractive index solvent and methyl naphthalene has one of the highest refractive indices for common solvents which are not too chemically hazardous.

Quartz silica is a ceramic material with a low refractive index, thereby achieving large cure depths at high solids

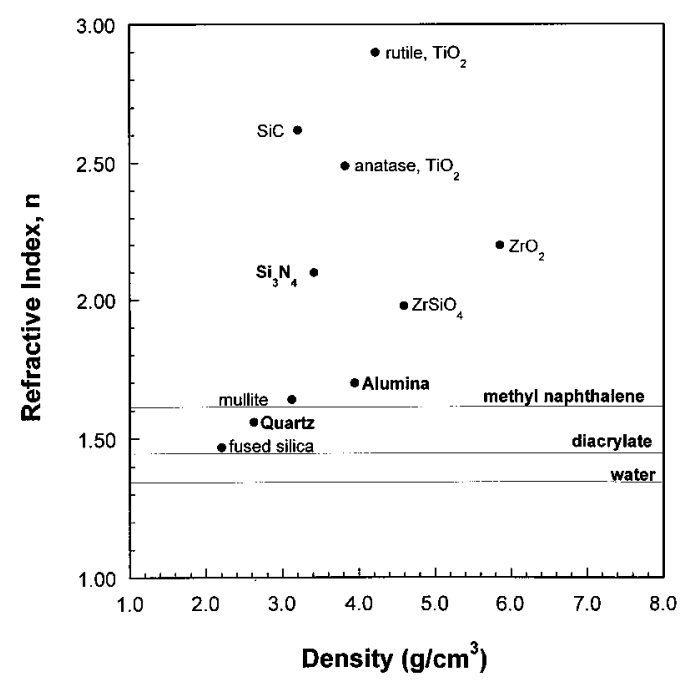

FIG. 8. Refractive index for a variety of ceramic materials in comparison with the refractive indices for two solvents (water and methyl naphthalene) and the diacrylate.

loadings when dispersed in aqueous UV-curable solutions. With silicon nitride acceptable cure depths $\left(D_{c}=200 \mu \mathrm{m}\right.$ stereolithography requirement) were not achievable due to the large refractive index difference between the ceramic and diacrylate. If the theoretical scattering equation is fit to the experimental data at 0.50 volume fraction solids so as to calculate the absolute cure depth $(40 \mu \mathrm{m})$, one can determine the refractive index needed to obtain a cure depth of $200 \mu \mathrm{m}$ at large doses. The value for the UV-curable solution would be 1.66, and methyl naphthalene is a good candidate. By tuning the refractive index difference, the cure depth can be modified to the user's choice.

\section{CONCLUSIONS}

The effective Beer's law equation [Eq. (2)] accurately describes the cure depth behavior for highly turbid, ultraviolet-curable suspensions, with linear proportional relationships for cure depth versus $1 / \phi$ and cure depth versus $\log \left(E_{0}\right)$. This validates the derivation of the scattering equation using Beer's law as a starting point.

For the extinction coefficient efficiency term $Q$, $Q=\widetilde{Q}\left(\Delta n^{2} / n_{0}^{2}\right)$, best represents the data as the factor controlling the depth of cure. Neither the Rayleigh-Gans nor Mie formulas predict the scattering behavior. With $Q=\Delta n^{2} / n_{0}^{2}$ inserted into the scattering equation, the magnitude of expected cure depth for any nonabsorbing ceramic powder dispersed in the aqueous or diacrylate solution can be predicted.

For turbid suspensions, scattering-limited cure depths do not appear to be simply related to particle size. Rather, the interparticle distance, $S$, appears to influence the resulting cure depth through the term $\widetilde{Q}$, where $\widetilde{Q} \propto S / \lambda$.

\section{ACKNOWLEDGMENTS}

We thank the sponsors, Dr. Steve Fishman and Dr. Ralph Wachter, at the Office of Naval Research for funding this work under contract N00014-913-1-0302. We are grate- 
ful to Dr. Paul Jacobs, Dr. Thomas Pang, and Kelly Kwo, at 3D Systems, in Valencia, CA, for their time and use of their stereolithography apparatus. The authors also wish to express their gratitude to the referee for the thorough reviews and helpful comments.

${ }^{1}$ C. E. Bohren and D. R. Huffman, Absorption and Scattering of Light by Small Particles (Wiley, New York, 1988).

${ }^{2}$ H. C. van de Hulst, Light Scattering by Small Particles (Wiley, New York, 1957).

${ }^{3}$ H. Jacobowitz, J. Quant. Spectrosc. Radiat. Transf. 11, 691 (1971).

${ }^{4}$ H. C. van de Hulst, Multiple Light Scattering: Tables, Formulas, and Applications, (Academic, New York, 1980), Vol. 2, Chap. 10.

${ }^{5}$ P. W. Barber and S. C. Hill, Light Scattering by Particles: Computational Methods (World Scientific, Teaneck, NJ, 1990)

${ }^{6}$ H. Blumer, Z. Phys. 32, 119 (1925).

${ }^{7}$ M. Kerker, The Scattering of Light and other Electromagnetic Radiation (Academic, New York, 1969).

${ }^{8}$ M. L. Griffith and J. W. Halloran, Manufacturing Science and Engineering, (American Society of Mechanical Engineers, New York, 1994), Vol. 68-2, pp. 529-534.

${ }^{9}$ M. L. Griffith and J. W. Halloran, Proceedings of the Solid Freeform Fabrication Symposium, Austin, TX, August 8-10, 1994, edited by H. Marcus et al. (University of Texas, Austin, 1994), pp. 396-403.

${ }^{10}$ M. L. Griffith and J. W. Halloran, Proceedings of the Sixth International Conference on Rapid Prototyping, Dayton, OH, June 4-7, 1995, edited by A. Lightman et al. (University of Dayton, Dayton, OH, 1995).

${ }^{11}$ M. L. Griffith, T-M Chu, W. Wagner, and J. W. Halloran, Proceedings of the Solid Freeform Fabrication Symposium, Austin, TX, August 7-9, 1995, edited by H. Marcus et al. (University of Texas, Austin, TX, 1995), pp. 31-38.

${ }^{12}$ C. Hull, U.S. Patent No. 4,575,330 (1986).

${ }^{13}$ P. F. Jacobs, Rapid Prototyping and Manufacturing: Fundamentals of Stereolithography, (Society of Manufacturing Engineers, Dearborn, MI, 1992).
${ }^{14}$ T. H. Pang, Proceedings of the Solid Freeform Fabrication Symposium, Austin, TX, August 8-10, 1994, edited by H. Marcus et al. (University of Texas, Austin, TX, 1994), pp. 204-224.

${ }^{15}$ M. L. Griffith and J. W. Halloran, J. Am. Ceram. Soc. 79, 2601 (1996).

${ }^{16}$ Michelle Lynn Griffith, Ph.D. thesis, University of Michigan, Materials Science and Engineering Department, March, 1995, Chap. 4 and 5.

${ }^{17}$ E. D. Pawlik, Handbook of Optical Constants of Solids, (Harcourt Brace Jovanovich, New York, 1985), pp. 749-764, 771-774.

${ }^{18}$ C. L. Haertling, S. Yoshikawa, and R. R. Newnham, J. Am. Ceram. Soc. 73, 3330 (1990)

${ }^{19}$ A. C. Young, O. O. Omatete, M. A. Janney, and P. A. Menchofer, J. Am. Ceram. Soc. 74, 612 (1991).

${ }^{20}$ O. O. Omatete, M. A. Janney, and R. A. Strehlow, Am. Ceram. Soc. Bull. 70, 1641 (1991).

${ }^{21}$ S. P. Pappas, Radiation Curing: Science and Technology, edited by S. P. Pappas, (Plenum, New York, NY, 1992), pp. 1-20.

${ }^{22}$ H. C. van de Hulst, Light Scattering by Small Particles, Sec. 2.6 (J. Wiley, New York, NY, 1957).

${ }^{23}$ H. C. van de Hulst, Light Scattering by Small Particles, (J. Wiley, New York, NY, 1957), Chap. 10 \& 11

${ }^{24}$ T. Allen, Particle Size Measurement, (Chapman and Hall, New York, 1990), pp. 491-494.

${ }^{25}$ R. J. Hunter, Introduction to Modern Colloid Science (Oxford Science, Oxford, 1993), pp. 80-87.

${ }^{26}$ C. E. Habermann, Encyclopedia of Chemical Technology, 4th ed. (1991), pp. 251-266.

${ }^{27}$ Chemistry of Acrylamide, Bull. PRC 109, Process Chemicals Department, American Cyanamid Co., Wayne NJ, 1969.

${ }^{28}$ C. E. Hoyle, Radiation Curing: Science and Technology (Plenum, New York, NY, 1992), pp. 273-299.

${ }^{29}$ Basic physics describes plane waves traveling through two widely separated slits that act as point sources for setting up constructive and destructive interference patterns.

${ }^{30}$ D. A. Weitz and D. J. Pine, Dynamic Light Scattering: The Method and some Applications, edited by W. Brown (Oxford University, Oxford, 1993), Chap. 16, pp. 652-720. 